International Journal of Pure and Applied Mathematics

Volume 98 No. 1 2015, 123-128

ISSN: 1311-8080 (printed version); ISSN: 1314-3395 (on-line version)

url: http://www.ijpam.eu

doi: http://dx.doi.org/10.12732/ijpam.v98i1.10

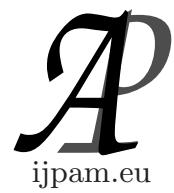

\title{
MONOIDS OF S-H FUZZY PARTITIONS OF A SET
}

\author{
D. Singh ${ }^{1}$, A.J. Alkali ${ }^{2}$ \\ ${ }^{1}$ Indian Institute of Technology Bombay \\ ${ }^{2}$ Department of Mathematics \\ Ahmadu Bello University, Zaria, NIGERIA \\ ${ }^{2}$ Department of Mathematics \\ Ahmadu Bello University Zaria \\ NIGERIA
}

\begin{abstract}
This research note constructs S-H fuzzy collections and S-H fuzzy partitions of a finite set. The main objective of the paper lies in defining two different operations on a class of $\mathrm{S}-\mathrm{H}$ fuzzy partitions of a set and, in turn, proving that these give rise to a monoid.
\end{abstract}

AMS Subject Classification: 03E72, 46S50, 06F05

Key Words: fuzzy sets, fuzzy partitions, monoid

\section{Introduction}

Zadeh [1] is known to be the pioneering work on extending the concepts of hard(i.e non-fuzzy) partitions and equivalence relations defined for finite sets to fuzzy partitions and similarity relations. Concurrently as well as subsequently, this endeavour of Zadeh attracted serious attention from a number of

Received: September 10, 2014

(c) 2015 Academic Publications, Ltd. url: www.acadpubl.eu

${ }^{\S}$ Correspondence author 
researchers, particularly [2], which also contains most extant related references. The most intriguing problem has been to justify transitivity fragment of similarity relation in the context of fuzzy sets. In the recent years, some works such as $[3,4]$ have appeared which describe fuzzy equivalence relations and partitions at par with their hard counterparts, and name it $S$-H fuzzy partition.

It is known that some important applications of the concept of equivalence, similarity, ordering, etc., defined for fuzzy sets, have been found in both pure and applied mathematics [1]. In particular, in view of the applications of certain monoids of partitions of a set in the areas of computer arithmetic, formal languages, and sequential machine [5], the objective of this paper centres at describing certain monoids of S-H fuzzy patitions of a set.

\section{Preliminaries}

Definition 1. (Fuzzy set [6]) A fuzzy set $\tilde{A}$ in a nonempty universe set $X$ is a function from $X$ into $[0,1]$. Let $\tilde{A}(x)$ denote the degree of $x$ in $\tilde{A}$. The fuzzy set $\tilde{A}$ is said to be contained in a fuzy set $\tilde{B}$ if and only if $\tilde{A}(x) \leq \tilde{B}(x), \forall x \in X$. The union of $\tilde{A}$ and $\tilde{B}$, denoted $\tilde{A} \cup \tilde{B}$, is defined by $(\tilde{A} \cup \tilde{B})(x)=\max [\tilde{A}(x), \tilde{B}(x)], \forall x \in X$. The intersection of $\tilde{A}$ and $\tilde{B}$, denoted $\tilde{A} \cap \tilde{B}$, is defined by $(\tilde{A} \cap \tilde{B})(x)=\min [\tilde{A}(x), \tilde{B}(x), \forall x \in X$. The compliment of $\tilde{A}$, denoted $\tilde{A}^{\prime}$, defined by $\left.\tilde{A}^{\prime}(x)=1-\tilde{A}(x)\right], \forall x \in X$.

Definition 2. (weak-separated fuzzy subsets [7]) Let $\tilde{F}$ be a collection of fuzzy subset of a nonempty set $X . \tilde{A}, \tilde{B} \in \tilde{F}$ with $\tilde{A} \neq \tilde{B}$. If $\mu_{\tilde{A} \cap \tilde{B}}(x)<$ $0.5, \forall x \in X$, then $\tilde{A}$ and $\tilde{B}$ are called weak-separated fuzzy subsets.

Definition 3. (Fuzzy Partition [3]) Let $X$ be a nonempty set. A fuzzy partition $\tilde{T}$ of $X$ is a set of nonempty fuzzy subsets of $X$ such that

(i) If $\tilde{A}, \tilde{B} \in \tilde{T}$ and $\tilde{A} \neq \tilde{B}$, then $(\tilde{A} \cap \tilde{B})(x)<0.5$, and

(ii) $\bigcup_{\tilde{w} \in \tilde{T}} \tilde{w}=X$.

Definition 4. (Weakly Empty Fuzzy Subset, [3]) Let $X$ be a nonempty set and $\tilde{A}$ be a fuzzy subset of $X$. $\tilde{A}$ is called a weakly empty fuzzy subset of $X$ if $\mu_{\tilde{A}}(x)<0.5, \forall x \in X$.

Definition 5. (Non-weakly empty fuzzy subset) A fuzzy subset of $X$ is called non-weakly empty fuzzy subset if it is not weakly empty. 
Definition 6. (S-H Fuzzy Collection [4]) Let $\tilde{F}$ be a collection of fuzzy subsets of a nonempty set $X$. $\tilde{F}$ is called a S-H collection if and only if $\tilde{B} \cap \tilde{A} \leq$ $\tilde{B}(a)$, whenever $\tilde{A}, \tilde{B} \in \tilde{F}$ such that $\tilde{A}(a)=1$.

Example 7. Let $X=\left\{x_{0}, x_{1}, x_{2}, x_{3}, x_{4}\right\}$ and let $\tilde{F}=\left\{\tilde{A}_{1}, \tilde{A}_{2}, \tilde{A}_{3}, \tilde{A}_{4}, \tilde{A}_{5}\right\}$ be a collection of fuzzy subsets of $X$ where

$$
\begin{aligned}
& \tilde{A}_{1}=\left\{\left(x_{0}, 1.0\right),\left(x_{1}, 0.9\right),\left(x_{2}, 0.8\right),\left(x_{3}, 0.7\right),\left(x_{4}, 0.1\right)\right\}, \\
& \tilde{A}_{2}=\left\{\left(x_{0}, 0.9\right),\left(x_{1}, 1.0\right),\left(x_{2}, 0.8\right),\left(x_{3}, 0.7\right),\left(x_{4}, 0.1\right)\right\}, \\
& \tilde{A}_{3}=\left\{\left(x_{0}, 0.8\right),\left(x_{1}, 0.8\right),\left(x_{2}, 1.0\right),\left(x_{3}, 0.7\right),\left(x_{4}, 0.1\right)\right\}, \\
& \tilde{A}_{4}=\left\{\left(x_{0}, 0.7\right),\left(x_{1}, 0.7\right),\left(x_{2}, 0.7\right),\left(x_{3}, 1.0\right),\left(x_{4}, 0.1\right)\right\}, \text { and } \\
& \tilde{A}_{5}=\left\{\left(x_{0}, 0.1\right),\left(x_{1}, 0.1\right),\left(x_{2}, 0.1\right),\left(x_{3}, 0.1\right),\left(x_{4}, 0.1\right)\right\} .
\end{aligned}
$$

It is immediate to see that $\tilde{A}_{i} \cap \tilde{A}_{j}(x) \leq \tilde{A}_{j}(x)$, where $\tilde{A}_{i}(x)=1, i=\overline{1,4}$ and hence, $\tilde{F}$ is a S-H collection. Moreover, $\tilde{A}_{5}$ is a weak empty fuzzy set, and

$$
\bigcup \tilde{A}_{i}=\left\{\left(x_{0}, 1\right),\left(x_{1}, 1\right),\left(x_{2}, 1\right),\left(x_{3}, 1\right),\left(x_{4}, 0.1\right) \neq X\right. \text {. }
$$

Also $\mu_{\tilde{A}_{1} \cap \tilde{A}_{2}}\left(x_{0}\right)=0.9>0.5$, and similarly for other such combinations.

It is to be noted that S-H collections may contain both weakly empty and non-weakly empty fuzzy subsets of a given set.

Definition 8. (S-H Fuzzy Partition [4]) Let $X$ be a nonempty set. An S-H fuzzy partition $\tilde{T}$ of $X$ is defined as a set of non- weakly empty fuzzy subsets of $X$ such that

(i) if $\tilde{A}, \tilde{B} \in \tilde{T}$ and $\tilde{A} \neq \tilde{B}$, then $(\tilde{A} \cap \tilde{B})(x)<0.5$ i.e., $\tilde{A}, \tilde{B}$ are weak separated fuzzy subsets,

(ii) $\bigcup_{\tilde{w} \in \tilde{T}} \tilde{w}=X$, and

(iii) $\tilde{T}$ is S-H collection. 


\section{Monoids of S-H Fuzzy Partitions of a Set}

Let $\tilde{T}$ be a S-H fuzzy partition of a set $X$ and, let $\tilde{A}_{i}(i=\overline{1, n})$ denote the blocks of the partition $\tilde{T}$. Let $\Pi(X)$ denote the collection of all S-H fuzzy partitions of $X$.

We define a binary operation $*$ on $\prod(X)$ as follows:

Given $\tilde{T}_{1}, \tilde{T}_{2} \in \prod(X)$, let $\tilde{T}_{1} * \tilde{T}_{2}$ be the fuzzy set consisting of all nonempty intersections of every block of $\tilde{T}_{1}$ with every block of $\tilde{T}_{2}$, viz.,

$$
\tilde{T}_{1} * \tilde{T}_{2}=\left\{\left\{\tilde{A}_{i} \cap \tilde{B}_{j}\right\}, i, j=\overline{1, n}\right\}
$$

where $\tilde{T}_{1}=\left\{\tilde{A}_{i}\right\}, \tilde{T}_{2}=\left\{\tilde{B}_{j}\right\}, \tilde{X}=\left\{\tilde{x}_{i}\right\}$ and $\tilde{A}_{i}, \tilde{B}_{j}, i, j=\overline{1, n}$, are given by $\left\{\left(x_{i}, \mu_{\tilde{A}_{\mathrm{i}}}\left(x_{i}\right)\right)\right\}$ and $\left\{\left(x_{i}, \mu_{\tilde{B}_{\mathrm{j}}}\left(x_{i}\right)\right)\right\}, \forall x_{i} \in X$, respectively.

It is immediate to see that the operation $*$ on $\prod(X)$ is both associative and commutative, since the operation $\cap$ on fuzzy sets is both associative and commutative and also every element $\tilde{T}_{k} \in \prod(X)$ is idempotent with respect to $*$ i.e., $\tilde{T}_{k} * \tilde{T}_{k}=\tilde{T}_{k}$. Moreover, the identity element with respect to $*$ is the $\mathrm{S}-\mathrm{H}$ fuzzy partition consisting of a single block. Thus $\left(\prod(X), *\right)$ is a commutative, idempotent monoid.

Similarly, if we define a binary operation $\circ$ on $\prod(X)$ such that every resulting fuzzy set consists of all non-empty union of every block of $\tilde{T}_{1}$ with every block of $\tilde{T}_{2}$, for all $\tilde{T}_{1}, \tilde{T}_{2} \in \Pi(X)$, then $(\Pi(X), \circ)$ also gives rise to a commutative, idempotent monoid with the partition consisting of singleton blocks as the identity element.

Example 9. Let $X=\left\{x_{0}, x_{1}, x_{2}, x_{3}\right\}, \tilde{T}_{1}, \tilde{T}_{2} \in \prod(X)$ such that $\tilde{T}_{1}=\left\{\tilde{A}_{1}, \tilde{A}_{2}, \tilde{A}_{3}, \tilde{A}_{4}\right\}$ and $\tilde{T}_{2}=\left\{\tilde{B}_{1}, \tilde{B}_{2}, \tilde{B}_{3}, \tilde{B}_{4}\right\}$, where $\tilde{A}_{i}, \tilde{B}_{i}, i=\overline{1,4}$, are as given below:

$$
\begin{aligned}
\tilde{A}_{1} & =\left\{\left(x_{0}, 1.0\right),\left(x_{1}, 0.4\right),\left(x_{2}, 0.3\right),\left(x_{3}, 0.2\right)\right\} \\
\tilde{A}_{2} & =\left\{\left(x_{0}, 0.4\right),\left(x_{1}, 1.0\right),\left(x_{2}, 0.3\right),\left(x_{3}, 0.2\right)\right\} \\
\tilde{A}_{3} & =\left\{\left(x_{0}, 0.3\right),\left(x_{1}, 0.3\right),\left(x_{2}, 1.0\right),\left(x_{3}, 0.2\right)\right\} \\
\tilde{A}_{4} & =\left\{\left(x_{0}, 0.2\right),\left(x_{1}, 0.2\right),\left(x_{2}, 0.2\right),\left(x_{3}, 1.0\right)\right\}
\end{aligned}
$$

and

$$
\begin{aligned}
& \tilde{B}_{1}=\left\{\left(x_{0}, 1.0\right),\left(x_{1}, 0.3\right),\left(x_{2}, 0.3\right),\left(x_{3}, 0.1\right)\right\} \\
& \tilde{B}_{2}=\left\{\left(x_{0}, 0.3\right),\left(x_{1}, 1.0\right),\left(x_{2}, 0.2\right),\left(x_{3}, 0.1\right)\right\} \\
& \tilde{B}_{3}=\left\{\left(x_{0}, 0.3\right),\left(x_{1}, 0.2\right),\left(x_{2}, 1.0\right),\left(x_{3}, 0.1\right)\right\} \\
& \tilde{B}_{4}=\left\{\left(x_{0}, 0.1\right),\left(x_{1}, 0.1\right),\left(x_{2}, 0.1\right),\left(x_{3}, 1.0\right)\right\}
\end{aligned}
$$


It is easy to verify that $\tilde{T}_{1}$ and $\tilde{T}_{2}$ are S-H fuzzy partitions of $X$.

Note that, $\tilde{A}_{i} \cap \tilde{B}_{j}$, for $i \neq j$ is weakly empty, for example,

$$
\tilde{A}_{1} \cap \tilde{B}_{2}=\left\{\left(x_{0}, 0.3\right),\left(x_{1}, 0.4\right),\left(x_{2}, 0.2\right),\left(x_{3}, 0.1\right)\right\} .
$$

We have, following the definition given above, for example,

$$
\tilde{T}_{3}=\tilde{T}_{1} * \tilde{T}_{2}=\left\{\tilde{A}_{1} \cap \tilde{B}_{1}, \tilde{A}_{2} \cap \tilde{B}_{2}, \tilde{A}_{3} \cap \tilde{B}_{3}, \tilde{A}_{4} \cap \tilde{B}_{4}\right\}=\left\{\tilde{C}_{1}, \tilde{C}_{2}, \tilde{C}_{3}, \tilde{C}_{4}\right\}
$$

where

$$
\begin{aligned}
& \tilde{C}_{1}=\left\{\left(x_{0}, 1.0\right),\left(x_{1}, 0.3\right),\left(x_{2}, 0.3\right),\left(x_{3}, 0.1\right)\right\}, \\
& \tilde{C}_{2}=\left\{\left(x_{0}, 0.3\right),\left(x_{1}, 1.0\right),\left(x_{2}, 0.2\right),\left(x_{3}, 0.1\right)\right\}, \\
& \tilde{C}_{3}=\left\{\left(x_{0}, 0.3\right),\left(x_{1}, 0.2\right),\left(x_{2}, 1.0\right),\left(x_{3}, 0.1\right)\right\}, \text { and } \\
& \tilde{C}_{4}=\left\{\left(x_{0}, 0.1\right),\left(x_{1}, 0.1\right),\left(x_{2}, 0.1\right),\left(x_{3}, 1.0\right)\right\} .
\end{aligned}
$$

Also, $\tilde{T}_{1} * \tilde{T}_{1}=\left\{\tilde{A}_{1} \cap \tilde{A}_{1}, \tilde{A}_{2} \cap \tilde{A}_{2}, \tilde{A}_{3} \cap \tilde{A}_{3}, \tilde{A}_{4} \cap \tilde{A}_{4}\right\}=\tilde{T}_{1} ; \tilde{T}_{1} * \tilde{T}_{2}=\tilde{T}_{2} * \tilde{T}_{1}$, etc. Similarly, results for various other combinations could be computed.

Thus $(\Pi(X), *)$ is a commutative, idempotent monoid with $\tilde{X}=\left\{\tilde{x}_{0}, \tilde{x}_{1}\right.$, $\left.\tilde{x}_{2}, \tilde{x}_{3}\right\}$, as the identity element.

It is intresting to see that another operation, denoted $\oplus$, can be defined on $\prod(X)$ such that $\left(\prod(X), \oplus\right)$ is also a monoid. We define $\oplus$ as follows: Let $X$ be a set and $\tilde{T}_{1}=\left\{\tilde{A}_{1} \tilde{A}_{2}, \tilde{A}_{3} \tilde{A}_{4}\right\}$ and $\tilde{T}_{2}=\left\{\tilde{B}_{1} \tilde{B}_{2}, \tilde{B}_{3} \tilde{B}_{4}\right\}$ be two S-H fuzzy partitions of $X$. A fuzzy subset $\tilde{T}$ of $X$ belongs to $\tilde{T}_{1} \oplus \tilde{T}_{2}$ if:

i) $\tilde{T}$ is the union of one or more elements of $\tilde{T}_{1}$,

ii) $\tilde{T}$ is the union of one or more elements of $\tilde{T}_{2}$,

iii) No fuzzy subset of $\tilde{T}$ satisfies i) and ii) except $\tilde{T}$ itself.

It follows that $\oplus$ is both associative and commutative. The fuzzy partition consisting of single elements of $X$ is the identity of the operation $\oplus$ on $\prod(X)$. For example, $\left\{\tilde{x_{0}}, \tilde{x_{1}}, \tilde{x_{2}}, \tilde{x_{3}}\right\}$ is the identity with respect to $\oplus$ on $\prod(X)$ in the example considered above. On the same lines, the algebraic structure defined by the other operation described above, could be illustrated.

\subsection{Concluding Remark}

This research note is a contribution towards developing a fragment of fuzzy algebras that could be applied in the areas in which its counterpart in set algebras has been applied [5]. We wish to indicate that developing a variant of extant algorithms ( See [8], for related reference) to compute monoidal structures, particularly involving complex objects such as fuzzy partitions, could be a challenging problem. 


\section{References}

[1] L. A. Zadeh, Similarity relations and fuzzy orderings, Information Science, 3(1971), 177-200. http://dx.doi.org/10.1016/S0020-0255(71)80005-1

[2] J. C. Bezdik, J. Douglas Harris, Fuzzy partitions and relations: An axiomatic basis for clustering, Fuzzy Sets and Systems, 1 (1978), 111-127. http://dx.doi.org/10.1016/0165-0114(78)90012-X

[3] M. A. Shakhatreh, W. A. Shatanawi, M. M. Jaradat, A.A. Fora, On fuzzy partitions, International Journal of Pure and Applied Mathematics, 30, No.4 (2006), 467-474.

[4] M. A. Shakhatreh, M. A. Hayajneh, S-H fuzzy partition and fuzzy equivalence relation, Int. J. Contemp. Math. Sciences, 5, No.8 (2010), 381-392.

[5] P. Tremblay and R. Manohar, Discrete Mathematical Structures with Applications to computer Science, The McGraw-Hill publishing company Limited, (1975).

[6] L. A. Zadeh, Fuzzy sets, Information Control, 8 (1965), 338-353. http://dx.doi.org/10.1016/S0019-9958(65)90241-X

[7] M. A. Shakhatreh, W-separated fuzzy subsets, Jour. Inst. Math. and Comp. Sci. (Math. Ser), 7, No.3(1994), 213-216.

[8] J. Bezdik, D. Harris, Convex decompositions of fuzzy partitions, J. Math. Anal. Appl., 67 (1979), 490-512. http://dx.doi.org/10.1016/0022247X(79)90039-8. 